\title{
Reasons for inadequate or incomplete imaging techniques
}

\author{
Cosmin Caraiani' ${ }^{1}$, Yi Dong², Anthony G. Rudd ${ }^{3}$, Christoph F. Dietrich ${ }^{4,5}$
}

\begin{abstract}
${ }^{1}$ Department of Medical Imaging, "Iuliu Hatieganu" University of Medicine and Pharmacy, Cluj-Napoca, Romania, ${ }^{2}$ Department of Ultrasound, Zhongshan Hospital, Fudan University, Shanghai, China, ${ }^{3}$ Division of Health and Social Care Research, King's College London, London, United Kingdom, ${ }^{4}$ Medizinische Klinik 2, Caritas-Krankenhaus Bad Mergentheim, Bad Mergentheim, Germany, ${ }^{5}$ Ultrasound Department, First Affiliated Hospital of Zhengzhou University, China
\end{abstract}

\begin{abstract}
Even if imaging has developed considerably during the last decades there still exist several factors which limit its capacities. These factors can either limit the usage of a technique or degrade images making them difficult to interpret. Magnetic resonance imaging (MRI) has, as an absolute contraindication, the presence of metallic devices marked as "MRI unsafe" and metallic foreign bodies close to the eye or vital structures. Claustrophobia and artefacts reduce the application and performance of MRI in a significant proportion of patients. The major disadvantages of computed tomography (CT) are the exposure to ionizing radiation inducing malignancies especially in pediatric patients and the risk of contrast induced allergies and nephropathy. Ultrasound is a safe, easily available and low-cost imaging technique without significant side effects for the patient. Obesity or bloating can severely limit ultrasound capacities.

This paper written by radiologists and clinicians, highlights the main reasons leading to inadequate imaging and points out solutions to avoid inaccurate diagnosis due to incomplete imaging or presence of artifacts.

Keywords: Magnetic Resonance Imaging (MRI); Computed Tomography (CT); Ultrasound (US); contraindications; artefacts
\end{abstract}

\section{Introduction}

Over the last few decades, advances in imaging have transformed the practice of medicine. The diagnostic process in almost every medical field would be inconceivable without imaging. Unfortunately, each imaging technique also has contraindications, limitations, and factors limiting its diagnostic potential. Imaging techniques can also be potentially harmful to the patient, as in the case of computed tomography (CT), where ionizing radiation is used.

Received 27.08.2018 Accepted 02.10.2018

Med Ultrason

2018, Vol. 20, No 4, 498-507

Corresponding author: Prof. Dr. med. Christoph F. Dietrich

Medizinische Klinik 2, Caritas Krankenhaus

Bad Mergentheim, Uhlandstr. 7,

D-97980 Bad Mergentheim, Germany

Phone: 49 (0)7931 - 58 - $2201 / 2200$

Fax: 49 (0)7931 - 58 - 2290

E-mail: Christoph.dietrich@ckbm.de
In this paper radiologists and clinicians focus on factors which make imaging techniques either unrealizable or degrade the image quality to a point in which they become uninterpretable. Progress in imaging techniques has also led to a significant decrease of incomplete or uninterpretable imaging procedures.

\section{Magnetic resonance imaging (MRI)}

MRI is a very useful and established imaging tool, which can give information of great diagnostic value. MRI was initially developed for neurological purposes but, as the technique progressed, it became an indispensable diagnostic tool in musculoskeletal [1], abdominal [2], and gynecologic imaging [3] MRI is increasingly used worldwide. It is estimated that in the last 10 years the number of MRI performed each year has increased by $20 \%$ [4]. Over 80 million MR exams are performed each year worldwide [5].

Unfortunately, in daily clinical practice, situations are encountered where MRI is not feasible or unable to provide the required amount of information. Such situations 
Med Ultrason 2018; 20(4): 498-507 499

can be classified into: 1) situations when MRI is not feasible due to claustrophobia or to absolute contraindications such as the presence of metallic foreign bodies or devices and 2) situations when MRI offers incomplete information due to inadequate imaging. The presence of metallic artifacts, uncontrolled breathing or patient movement can lead to image degradation. Lack of patient comfort due to a closed tube or to high acoustic noise may also lead to decrease in image quality. Factors related to the machine or the technique used may limit MRI's potential of offering useful information. Contrast media administration must be avoided in some circumstances, but non-administration of contrast media may diminish the amount of useful information.

\section{1) Situations when MRI is "non-feasible"}

\section{Foreign bodies or medical implants which} represent an absolute contraindication to MRI

Materials used as implants and devices are classified as "MRI safe", "MRI conditional", or "MRI unsafe" [6]. Patients with MRI safe devices can be scanned without concern, but it should be noted that this terminology refers only to potential side effects that could produce harm to the patient; it does not guarantee that the presence of "MRI safe" material will not degrade image quality. In practice, a patient with an "MRI safe" device such as a hemostatic clip close to a rectal tumor or fistula can result in an examination that is uninterpretable because of artefacts (fig 1).

The presence of "MRI conditional" devices means that MRI is feasible, under some circumstances, while the presence of "MRI unsafe" materials represents a absolute contraindication. The conditions under which patients with materials considered as "MRI conditional" can be imaged are required to be stated by the manufacturer [7]. As a general rule, practitioners should never assume the MR compatibility of a device if it is not clearly documented in writing. Even if most modern devices are designed to be MRI-safe, the safety of MRI should not be assumed only on the basis that the implanted device was manufactured recently [8].

A device can be "MRI conditional" on some MRI machines and "MRI unsafe" on other machines. Factors related to the MRI examination such as strength of the magnetic field, maximum spatial gradient magnetic field of the magnet or pulse-sequence parameters can vary significantly among different MR systems leading to variable tolerance of implanted devices. Information on the safety of implants and devices can be obtained on the site www.MRIsafety.com [9].

The presence of MRI unsafe materials is a concern to the health system as many patients with MRI unsafe

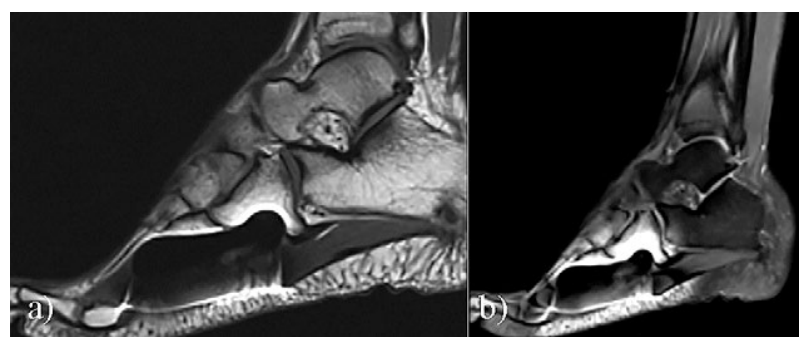

Fig 1. MRI of the foot of a football player with an ancient, treated metatarsal fracture: Even if the osteosyntetic material was marked as "MRI-safe" images obtained at the site of the fracture are uninterpretable.

materials need to undergo an MRI during their lifetime. Most often, in daily practice, the following devices are MRI unsafe: pacemakers and implantable cardioverterdefibrillators (ICD), implanted neurostimulators, brain aneurysm clips made from ferromagnetic material, hemodynamic monitoring and temporary pacing devices; hemodynamic support devices. The frequency with which these devices are present in those who require an MRI during their lifetime has led to a reconsideration of contraindications, ex: 2 million US citizens and 6 million worldwide have 'MRI unsafe' pacemakers. A recent multicenter study performed on patients with "non-MRI conditional" cardiac pacemakers and ICD's on 1.5T machines involved 1500 patients and no deaths, ventricular arrhythmias, device or lead failure were noted. Of note in this study all devices were reprogrammed before MRI and no MRI's of the thoracic region were undertaken [10]. However, MRI of such patients, if absolutely needed, has to be performed in experienced centers under very strict conditions of surveillance and machine settings. A large population based study estimated that up to $84 \%$ of patients with neurostimulation devices (which are, in their vast majority, marked as MRI unsafe) will have indications for an MRI scan during the following 5 years, data which can be somehow extrapolated also to patients with pacemakers or ICD's [4].

Metal fragments near the eye or great vessels

Shifting of metallic foreign bodies under the influence of MRI can lead to damage to vital structures such as nerves, vessels, or the eye. If there is any doubt about the presence or location of a metallic foreign body, an $\mathrm{X}$-ray should be performed prior to the MRI [11]. Surgery to remove the foreign body may be needed to safely perform MRI.

In particular cases, when information obtained by MRI is very important for the patient, MRI can be done despite the presence of presumed ferromagnetic foreign bodies - but only if they are not located in or near a vital structure. 


\section{Anxiety and claustrophobia}

Even if it is not a contraindication for MR imaging, claustrophobia and anxiety related reactions may lead to failure of MR imaging. Anxiety due to MRI was comparable, to anxiety before surgery [12]. The rate reported for failed examination because of anxiety varies in the literature between 0.7 and $20 \%[13,14]$. It has been reported that $4 \%-30 \%$ of the population suffered from anxiety symptoms during MR examinations and that $3 \%-5 \%$ of patients were unable to complete MR examinations [14]. Most of the papers refer only to patients who are unable to complete their MR procedure due to development of anxiety and claustrophobic symptoms, but did not include patients who completely refuse MRI. One study found that $10.6 \%$ of woman refused breast MRI due to fear of claustrophobia symptoms [15]. The percentage of patients unable to complete MR procedures can be reduced to only $0.7 \%$ of patients by using good communication techniques, quieter modern scanners with a shorter bore and reducing scanning times to a minimum. Open scanners can also significantly contribute to a reduction in claustrophobia sensations [16]. Modern scanners are open at both ends which offer the option of scanning the patient head-first. Claustrophobic reactions may also be minimized by sedating the patient before the procedure. Despite all these options a further $0.96 \%$ of patients, will have uninterpretable images due to motion artefacts even if they are able to finish the MR examination [17].

\section{2) Situations when MRI is feasible but difficult to interpret/uninterpretable}

\section{Presence of artefacts}

\section{(related to metallic objects or motion)}

The presence of artefacts can lead to poor quality images and difficult interpretation (fig 2).

Metallic artefacts can lead to non-visualization or bad visualization of a body part (fig 3 ). Methods for reducing the amplitude of metallic artifacts will vary from simple to complex. Some of the simple methods are: a high readout bandwidth; swapping frequency and phase; using thin sections; lower magnet strength (artifacts are more significant at $3.0 \mathrm{~T}$ compared to $1.5 \mathrm{~T}$ ); increase the matrix; increase the number of excitations (NEX); shorter echo-spacing; the use of metal correction artifact software such as MAVRIC or SEMAC $[18,19]$.

The cooperation of the patient is needed if MRI is to be properly performed, with the patient being required to remain still during the examination (the duration of such an MRI examination can be up to 30-40 min). Respiratory motion is also a major cause of artefacts in abdominal and thoracic imaging. Some sequences require the patient to hold their breath for almost $20 \mathrm{~s}$, a feat that is practical-

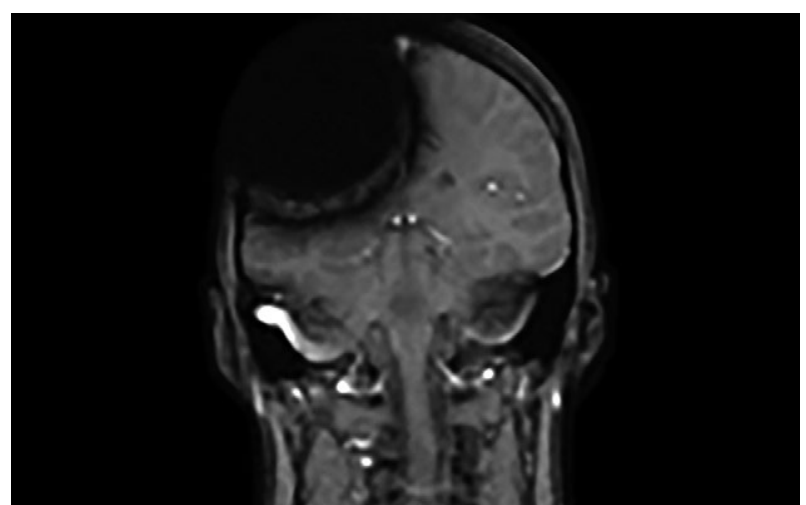

Fig 2. Brain MRI of a young patient. Severe artifacts due to the presence of a hairclip. Images of the right cerebral hemisphere are uninterpretable. The examination was continued after removal of the hairclip.

ly impossible in severely ill and sedated patients. Either breath-hold techniques or free-breathing with respiratory gating can be used to avoid respiratory related artefacts [20]. If gated acquisitions are to be efficient and artefacts avoided, techniques involving free-breathing require the patient to breath in a regular manner. Faster sequences can also reduce the amplitude of breathing artefacts. Before improvements in MR techniques, including the use of faster sequences, $5 \%$ of abdominal MRI were severely degraded due to breathing artefacts. This figure has now dropped to $0.5-1 \%[21,22]$.

There are a series of measures which can be taken to minimize motion artifacts: stabilization of the patient; using surface coils confined to the area of interest; using

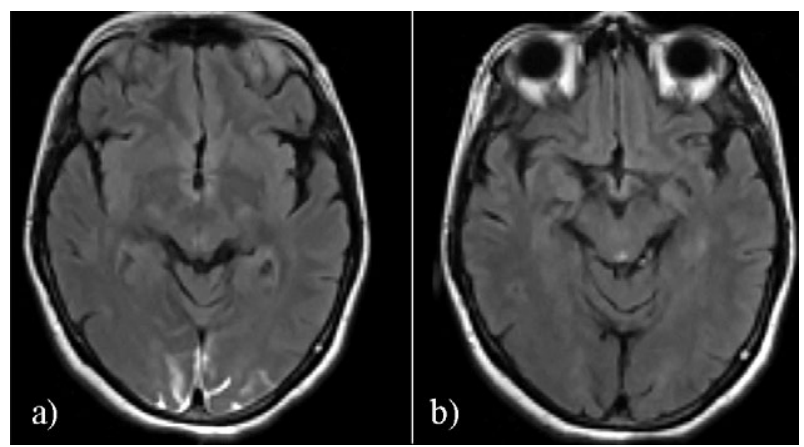

Fig 3. Brain MRI of a 55-years old female patient with mild headaches in her medical history, asymptomatic in the moment of the examination. Image 3a shows hyperintensity of the bilateral posterior cerebral sulci on the Flair sequence, an aspect which is highly suspicious for subarachnoid hemorrhage. Due to the contradiction between the imaging aspect and the patients good condition she was recalled for an repeat examination which proved to be normal (Image $3 b$ ). The aspect in $3 \mathrm{a}$ was due to artifacts from a hairclip which was not removed by the radiographer. 
spatial saturation pulses; flow saturation pulses; increasing number of signals averaged; swapping frequency and phase encoding directions; use of single-slice ultrafast sequences; using self-correcting sequences like PROPELLER and BLADE.

\section{3) Administration of Gadolinium based contrast agents (GdCA)}

\section{Allergic reactions to Gadolinium contrast agents}

The rate of acute allergic reactions after administration of GdCA is low, $0.07 \%-0.7 \%$, with anaphylaxis due to GdCA occuring in approximately 1:100 000 patients $[23,24]$. The side effects of GdCA are generally mild and include headache, vomiting, nausea, rash, local burning and thrombophlebitis at the site of the injection [25]. Patients with previous allergic reactions to Gadolinium are at a higher risk of developing adverse reactions to subsequent administration of GdCA. Injection of contrast media in such patients should therefore be avoided or, if there is an absolute need for injecting contrast media, patients should be premedicated with corticosteroids and/or antihistamines [23].

\section{Nephrogenic systemic fibrosis (NSF)}

NSF is a rare life-threatening disease that affects patients with renal failure who have had GdCA administered; that is why patients with a glomerular filtration rate (GFR) $>30 \mathrm{ml} / \mathrm{min}$ should not receive these contrast agents. Contrast agents may be classified as high risk (gadopentetic acid), medium risk (gadobenic acid) or low risk (gadoteridol) [7], 78\% of NSF being associated with the administration of Omniscan (gadodiamide) and 20\% with Magnevist (gadopentetate dimeglumine). Virtually no cases of NSF have been reported after the administration of MultiHance or Eovist [26,27]. Recent data suggest that class II Gd-based contrast agents can be safely administrated in patients with impaired renal function. Precautions still need to be taken and the lowest possible quantity of contrast necessary for the diagnosis should be injected.

\section{Computed tomography (CT)}

CT has, over MRI, the advantages of being more easily available and quick to perform. The ability to scan multiple regions and organs in a single examination and in very short period of time (1-3 min) makes CT the technique of choice for the follow-up of oncological patients. $\mathrm{CT}$ is also indicated in abdominal, thoracic, neurological, skeletal pathology and in traumatic and non-traumatic emergencies [28].

There are no absolute contraindications for unenhanced CT examination, although there is still a patient risk due to radiation exposure. According to the ALARA ("as low as reasonably achievable") principles, exposure to the patients must be minimal. Every time a procedure using ionizing radiation can be substituted with a nonionizing procedure allowing the same amount of information to be obtained and having the same diagnostic benefit, the non-irradiant procedure should be used. In order to avoid unnecessary irradiation, the radiologist must discuss, on a case-by-case basis, the appropriateness of CT scanning.

$\mathrm{CT}$ is in some cases inadequate. This may be due to the presence of artefacts. Non-administration of contrast media will decrease the amount of information which can be obtained and there are strong relative contraindications to contrast media administration.

\section{1) Avoiding unnecessary irradiation of patients}

Abdomino-pelvic CT is a relatively high-radiation dose examination in comparison with chest and head CTdue to the large number of radiosensitive organs in the field of view (imparts a dose of 6-10 $\mathrm{mSv}$ to the adult patient [29]). The mean dose in abdominal CT ( $8 \mathrm{mSv})$ corresponds to approximately 11 conventional abdominal radiographs $(0.7 \mathrm{mSv})$, which is equivalent to $3-4$ years of natural background radiation [30]. In the United States, radiation exposure from radiological procedures grew from $0.54 \mathrm{mSv}$ per person in 1980 to $3.0 \mathrm{mSv}$ per person in 2006, particularly due to the development of CT. In 2006, CT accounted for one half of all radiation exposure, despite accounting for only $17 \%$ of all imaging procedures [31].

Many low-radiation dose protocols have been developed, although most of them also lead to reduced image quality and conspicuity $[32,33]$. Such protocols are particularly useful in patients $<40$ years or in conditions in which fine detail is not needed, such as in nephrolithiasis. Also, the number of scans before and after contrast media injection has to be limited to a minimum necessary. Guidelines, such as the American College of Radiology (ACR) Appropriateness Criteria, should be used to choose the most appropriate imaging procedure and reduce unnecessary irradiation.

A large population based survey showed that $56 \%$ of the medical tests which are overordered are imaging procedures, mostly CT-scans [34]. The use of a Web-based computerized radiology entry system based on the ACR appropriateness criteria have reduced by $3 \%$ the outpatient CT procedure volume in a single institution [35]. Different papers suggests that $\mathrm{CT}$ is especially overused in clinical scenarios such as the diagnosis of minor head trauma [35] (37\% of patients have no indication) and the diagnosis of appendicitis [36]. 


\section{2) Situations in which $C T$ is indicated but} it provides incomplete information

\section{Artefacts in CT and uninterpretable CT images}

Artefacts can seriously degrade the quality of CT images, in some cases to the point of making them uninterpretable. Fortunately, the incidence where image alteration is so profound that interpretation is impossible is low and useful information can be obtained, even from an imperfect examination.

Artefacts can be classified into physics-based artefacts such as beam-hardening, which are related to the process of image acquisition, patient-related artefacts due to patient movement or metallic objects inside or on the skin of the patient and scanner-based artefacts related to imperfections in scanner functioning [37]. Most of the physics and scanner-based artefacts can be avoided or minimized by the correct use of the technique or by using the software available on new scanners to improve image quality and conspicuity.

Metal artefacts are extremely common, having been documented as affecting up to $10 \%$ of scans in one series, although most of them have no major impact on image visualization [38]. However, in some cases the presence of metallic objects can lead to severe streaking artefacts. To avoid these, patients should be asked to remove pieces of jewelry before the procedure. Furthermore, medical objects such as wires or leads should be removed from the body before the procedure. Gantry angulations can sometimes be used to exclude non-removable objects from the scanned field. If it is not possible to exclude metallic objects from the scanned field, it may still be possible to obtain interpretable images by increasing the kilovoltage and obtaining thin sections; corrections which unfortunately also lead to an increase in radiation dose. In addition, the impact of artefacts on image quality can be reduced by applying software corrections during the post-processing of the images [37]. In recent years, new post-processing techniques have been used in order to reduce metallic artefacts (MAR- metal artefact reduction techniques). They have different commercial names depending on the different vendors- OMAR (Philips), SEMAR (Toshiba) or MARS (General Electric) [39]. The reported reduction of noise by using these softwares is between 53 and $77 \%$.

Motion can cause blurring and double images, as well as long streaks. Faster scanners have considerably reduced motion artefacts [40].

Cardiac CT is particularly prone to motion artifacts and as a result frequently leads to uninterpretable images. With the increase in speed of the scanners there has been a decrease in the number of uninterpretable exams. Origi- nally with 4-slice scanners the percentage of uninterpretable studies was 20 and $40 \%$ but with the use of 64 and 128 slice scanners less than $3 \%$ of scans are uninterpretable $[41,42]$.

Contraindications to contrast media administration

Contrast media administration is particularly useful in abdominal pathology. In contrast to chest CT, where the presence of air and bone provides a natural contrast, most abdominal structures have a similar soft tissue density, so differentiating between them can be difficult on non-enhanced scans. Additionally, the visualization and correct characterization of focal liver lesions requires administration of contrast media [43].

Allergy to iodinated contrast media is rare, 0.2 to $0.6 \%$ of patients developing allergic reactions and $0.04 \%$ developing severe allergic reactions $[44,45]$. Nearly all life-threatening reactions to contrast media occur in the first 20 minutes after injection.

Patients with prior allergy to contrast media have 5-times greater risk of developing allergic reactions when contrast media is injected a second time. One recent study estimated that $19.5 \%$ of patients with an initial moderate to severe allergic reaction to contrast media will develop allergic reactions when contrast media is reinjected [46]. Patients with unrelated allergies have 2-3-fold higher risk of developing allergic reactions to contrast media. The use of non-ionic contrast media has contributed to a significant decrease in adverse reactions during contrast media administration [47]. Infants and the elderly have lower risk of developing allergic reactions to contrast media administration comparing with middle-aged people. The pretesting of patients to contrast media does not predict which patients are prone to allergic reactions, and does not lower the incidence of contrast media reactions. In a meta-analysis of over 300000 cases, the predictive value and sensitivity of pretesting for anticipating the reaction to contrast media were extremely low, with values of $1.2 \%$ and $3.7 \%$ respectively [48]. Studies have shown that a test-reaction to contrast media can actually increase the incidence of contrast media reactions [49]. Mortality due to low-osmolarity contrast media is extremely low. A large Japanese study described no fatal reactions in 170000 injections [47].

History of a prior allergy to contrast media is considered a strong relative contraindication to contrast media administration. If contrast media is absolutely necessary for diagnostic purposes, it can be performed after premedication with corticosteroids and antihistamine drugs. Changing the contrast medium has also lowered the incidence of repeated allergic reactions in patients with prior allergic reactions to contrast media administration [50]. Routine premedication for patients with other types of 
allergies is not indicated. Contrast media administration should not be withheld in patients with allergies, other than those to iodinated contrast media.

Even if renal insufficiency is a strong relative contraindication for contrast media administration anuric patients in end-stage renal disease with no transplanted kidneys can have contrast media safely.

\section{3) Challenges of CT and MRI of the obese patient}

Severe obesity can also be a limitation for CT and MRI. Most CT and MRI machines cannot accommodate patients with a weight above $160 \mathrm{~kg}$ or an abdominal diameter of more than $60 \mathrm{~cm} \mathrm{[51],} \mathrm{although} \mathrm{some} \mathrm{modern}$ machines can exceed these limits up to $190-200 \mathrm{~kg}$. For both techniques, if patients fit into the gantry the images will generally be diagnostic. Large-weight capacity CT and MRI machines capable of imaging patients heavier than $200 \mathrm{~kg}$ are on the market, but only $10 \%$ of emergency units in the United States have such machines [52]. Approximately $2 \%$ of emergency patients in the US have a weight of more than $160 \mathrm{~kg}$ and one quarter of CT scans and two thirds of MRI machines are not able to image such patients. Another paper found that $2 \%$ of ultrasound studies, $0.4 \%$ of abdominal MRIs, and $0.1 \%$ of abdominal CTs are "limited by the body habitus" in obese patients, which means they are inadequate. This does not include patients who cannot be imaged by CT or MRI due to excessive body weight exceeding the weight-bearing capacity of the table [53]. In obese patients, CT has to be used with caution, because the dose of irradiation is higher than in normal weight patients; an increase in weight of $40 \%$ will elevate the dose of irradiation by $100 \%$ [54].

\section{Ultrasound (US)}

US is a very useful and well-established tool for abdominal imaging. US is readily available, comparatively inexpensive, can be used at the bedside and is easily accepted by the patient. In comparison with CT and MRI it has the advantages of safety, low-cost, and availability and can be repeated as often as necessary. It provides valuable information regarding the abdominal solid viscera and is an excellent technique for real time depiction of the presence of fluid in the peritoneal or pleural spaces.

US is also a useful tool in other fields of pathology, such as soft tissue pathology, gynecology and obstetrics. The pathology of thyroid, salivary glands, cervical lymph nodes and the eye can also be characterized by means of US. US can also serve in guiding therapeutic and diagnostic procedures.

US has no absolute contraindications even if there exist precautions to be taken in the first trimester of preg- nancy: exposure time has to be limited at a minimum, mechanical and thermal indices have to be set as low as possible and Doppler ultrasound is to be avoided [55]. The use of US during pregnancy for non-medical purposes such as obtaining fetal videos has been discouraged.

\section{1) Difficulties and sources of error in ultrasound}

As with other diagnostic methods, errors may occur in ultrasound examinations [56] due to multiple factors: errors caused by the US machine, choice of transducers or setting (inadequate equipment quality); errors caused by the patient's condition or preparation for the exam (e.g. in patients who cannot hold a breath for a couple of seconds, organs located behind the costal cage such as the liver or spleen may be difficult to examine; in patients who have eaten before ultrasound, it may not be possible to visualize the pancreas); failure to obtain an optimal image of the tested organs (in Chilaiditi syndrome parts of the liver may not be visualized due to interposition of the transverse colon; in some patients, parts of the pancreas cannot be seen on ultrasound). Meteorism is a concern for abdominal ultrasound.

A retrospective study focusing on ultrasound of cirrhotic livers for the detection of hepatocellular carcinoma (HCC) found that $20 \%$ of screening USs were of inadequate quality [57]. Body mass index and male gender were the strongest predictive factors for inadequate imaging, with the most common reasons being rib shadowing and inadequate beam penetration resulting in visualization of less than two thirds of the liver. A severely shrunken liver in advanced cirrhosis is difficult to descend below the rib cage.

\section{2) US and obesity}

US is the imaging technique most affected by obesity, because of the depth of penetration required with resultant attenuation of the ultrasound beam [51]. In the imaging of an obese patient with $8-\mathrm{cm}$ of subcutaneous fat, $94 \%$ of the original sound wave is attenuated before it reaches the peritoneal cavity [58]. A retrospective study of over 5000000 imaging studies found that $1.5 \%$ of abdominal US examinations were unsatisfactory due to obesity. This number is increasing with the increase in the prevalence of obesity. In 1989 , only $0.7 \%$ of abdominal ultrasounds were considered unsatisfactory due to obesity; this percent rose to $1.9 \%$ in 2003 [59].

There are some techniques available that can improve the visualization of peritoneal organs in obese patients, including the use of the lowest frequency of a multifrequency transducer (the "penetration" mode) and harmonic imaging, which reduces the deleterious effects of subcutaneous tissues [60]. The signal-to-noise ratio can 
Table I. Lists with factors which lead to inadequate imaging and solutions to obtain correct images in these patients

\begin{tabular}{|c|c|c|c|c|}
\hline $\begin{array}{l}\text { Imaging } \\
\text { technique }\end{array}$ & $\begin{array}{l}\text { Factors which make } \\
\text { the examination } \\
\text { unrealizable }\end{array}$ & $\begin{array}{l}\text { Factors which lead to } \\
\text { image degradation }\end{array}$ & $\begin{array}{l}\text { Percentage of } \\
\text { patients affected }\end{array}$ & What to do in these circumstances \\
\hline \multirow[t]{7}{*}{ MRI } & Claustrophobia & & $1-2 \%$ & $\begin{array}{l}\text { Using open scanners } \\
\text { Sedation of patients } \\
\text { Reducing the noise }\end{array}$ \\
\hline & $\begin{array}{l}\text { Presence of metallic } \\
\text { implants, devices or } \\
\text { foreign bodies }\end{array}$ & & $\sim 2.7$ USA citizens & $\begin{array}{l}\text { Removal of the foreign body (if ferromag- } \\
\text { netic) } \\
\text { Using MR conditional or MR safe devices }\end{array}$ \\
\hline & & $\begin{array}{l}\text { Metallic implants or } \\
\text { devices which are not } \\
\text { absolute contraindication } \\
\text { to MRI }\end{array}$ & $0.5-1 \%$ & $\begin{array}{l}\text { Using a high readout bandwidth } \\
\text { Swapping frequency and phase } \\
\text { Using thin sections } \\
\text { Lower magnet strength. Artifacts are more } \\
\text { important at } 3.0 \mathrm{~T} \text { as compared to } 1.5 \mathrm{~T} \\
\text { Increase the matrix } \\
\text { Increase the number of excitations NEX } \\
\text { Shorter echo-spacing }\end{array}$ \\
\hline & & $\begin{array}{l}\text { Factors related to the } \\
\text { patient (breathing or } \\
\text { moving during the ex- } \\
\text { amination) }\end{array}$ & & $\begin{array}{l}\text { Stabilization of the patient } \\
\text { Using surface coils confined to the area of } \\
\text { interest } \\
\text { Using spatial saturation pulses } \\
\text { Flow saturation pulses } \\
\text { Increasing number of signals averaged } \\
\text { (NEX) } \\
\text { Swapping frequency and phase encoding } \\
\text { directions } \\
\text { Use of single-slice ultrafast sequences } \\
\text { Using self-correcting sequences like PRO- } \\
\text { PELLER and BLADE }\end{array}$ \\
\hline & & $\begin{array}{l}\text { Factors related to the } \\
\text { machine or the technique } \\
\text { used }\end{array}$ & $\begin{array}{l}\text { MR accounts } \\
\text { for }>90 \% \text { recalls } \\
\text { among imaging } \\
\text { techniques }\end{array}$ & $\begin{array}{l}\text { Good quality and maintenance of MRI } \\
\text { machines. } \\
\text { Using highly-qualified MR technicians }\end{array}$ \\
\hline & & $\begin{array}{l}\text { Contraindications to } \\
\text { contrast media adminis- } \\
\text { tration }\end{array}$ & $0.17-2.8 \%$ & $\begin{array}{l}\text { Premedication of patients; changing the } \\
\text { type of contrast agent }\end{array}$ \\
\hline & Morbid obesity & & $2-3 \%$ in the USA & Use of large weight capacity scanners \\
\hline \multirow[t]{5}{*}{$\mathrm{CT}$} & $\begin{array}{l}\text { Limiting irradiation in } \\
\text { patients where diag- } \\
\text { nostic benefit of CT is } \\
\text { low or when CT can be } \\
\text { replaced with another } \\
\text { imaging technique }\end{array}$ & & $\begin{array}{l}\text { Using correct and } \\
\text { appropriate indica- } \\
\text { tions will reduce } \\
\text { the number of CT- } \\
\text { scans by } 3 \%\end{array}$ & $\begin{array}{l}\text { Correct choice of the imaging technique. } \\
\text { Using CT only when it is unavoidable } \\
\text { Using low-dose protocols }\end{array}$ \\
\hline & & $\begin{array}{l}\text { Metallic implants or } \\
\text { foreign bodies leading to } \\
\text { image degradation }\end{array}$ & & $\begin{array}{l}\text { Remove metallic objects Usage of post- } \\
\text { processing techniques such as Metal Dele- } \\
\text { tion Techniques }\end{array}$ \\
\hline & & $\begin{array}{l}\text { Factors related to the } \\
\text { patient (breathing or } \\
\text { moving during the ex- } \\
\text { amination) }\end{array}$ & $\begin{array}{l}<3 \% \text { of cardiac } \mathrm{CT} \\
\text { exams are unin- } \\
\text { terpretable due to } \\
\text { cardiac motion }\end{array}$ & $\begin{array}{l}\text { Using faster scanners } \\
\text { Sedation of patients } \\
\text { Use of beta-blockers to reduce the heart } \\
\text { frequency and variability (for cardiac CT). } \\
\text { ECG-editing }\end{array}$ \\
\hline & & $\begin{array}{l}\text { Contraindications to } \\
\text { contrast media adminis- } \\
\text { tration }\end{array}$ & $\begin{array}{l}0.2-0.6 \% \text { of } \\
\text { patients develop } \\
\text { mild or moderate } \\
\text { allergic reactions to } \\
\text { contrast media; }\end{array}$ & $\begin{array}{l}\text { Using an alternative imaging technique or } \\
\text { premedication for patients with prior aller- } \\
\text { gies to contrast media }\end{array}$ \\
\hline & Morbid obesity & & $2-3 \%$ in the USA & Use of large weight capacity scanners \\
\hline
\end{tabular}




\begin{tabular}{lll}
\hline Ultrasound & $\begin{array}{l}\text { Uncooperative patients } \\
\text { Obesity }\end{array}$ & $\begin{array}{l}\text { Sedation } \\
\text { Reducing the frequency of the ultrasound } \\
\text { beam }\end{array}$ \\
Bloating & $\begin{array}{l}\text { Using tissue harmonics } \\
\text { Dietary restrictions } \\
\text { Physical exercise } \\
\text { Premedication }\end{array}$ \\
\hline
\end{tabular}

MRI - magnetic resonance imaging; CT - computer tomography; USA - United States of America

also be improved by the use of compound imaging and the presence of speckle reduction filters [61]. In pelvic ultrasound, a transvaginal or transrectal approach can improve visualization.

\section{3) Contrast-enhanced ultrasound (CEUS)}

The main indication for CEUS is characterization of focal liver lesions (FLL). The quality of the image on CEUS depends on the quality of the image on US; when the patient is obese or bloated or the lesion is too profound, CEUS will be inadequate [62]. In a large multicenter study, CEUS had sufficient diagnostic quality to allow assessment of 1280 out of 1349 focal liver lesions (94.9\%). The remaining $5.1 \%$ of FLL could not be appropriately characterized because of adiposity of the patient, steatosis of the liver, the presence of calcifications or air interference [63].

\section{General considerations}

Even if imaging has shown substantial progress over the last few decades, diagnostic limitations still exists for each technique. With $33 \%$ of American adults being obese, a percentage is increasing, obesity is a major public health hazard and is influencing the ability to image pathology in these patients [60]. Obese patients present limitations for the use and diagnostic capabilities of US, $\mathrm{CT}$ and MRI. Furthermore, 3\% of American adults are considered morbidly obese, and imaging them can be extremely difficult, sometimes only being possible in centers with dedicated CT and MRI machines [64]. Also, obese patients tend to show greater mortality from cancer than normal weight patients. This may be due to difficulties in both diagnosis and treatment of cancer in obese patients [65].

The imaging of patients with chronic renal disease and a GFR lower than $30 \mathrm{ml} / \mathrm{min} / 1.73 \mathrm{~m}^{2}$ is challenging, as administration of either iodinated contrast media or gadolinium products is a relative contraindication. Administration of Sonovue is not contraindicated in these patients, and CEUS can represent an alternative diagnostic tool; for example, in characterization of FLL where contrast injection is mandatory. Furthermore, recent FDA regulations have requested that only Omniscan, Magnevist and Optimark should be contraindicated in the setting of renal insufficiency. If contrast administration is absolutely necessary to obtain a correct diagnosis, the radiologist should inject one of the other GdCA available on the market (Ablavar, Dotarem, Eovist, Gadavist, MultiHance or Prohance) at the lowest sufficient dose [66].

MRI is an imaging technique performed in an unfriendly environment due to the closed scanner bore and the presence of acoustic noise. This environment leads to increased anxiety, particularly in patients with head injuries, psychiatric disorders, the elderly and infants [67]. However, anxiety leading to premature termination of examinations and consequently to inadequate imaging, can affect patients regardless of their age, gender or preexisting psychiatric pathology. Recent studies have demonstrated decreases in both claustrophobic symptoms and premature termination of exams with the use of modern MRI scanners with a shorter and wider bore and reduced acoustic noise (from 3-5\% to 1-2\%). However, many studies underestimate the number of patients unable to perform MRI, because they do not include those patients who completely refuse it.

Motion artifacts (e.g. in uncooperative patients) can lead to image degradation and to uninterpretable examinations in both CT and MRI. If patients cannot be sedated, then $\mathrm{CT}$ could be a better option because it is quicker to perform and less prone to artifacts.

Metallic artifacts can affect both techniques, with MR examinations once again being more influenced, and sometimes being rendered uninterpretable. Modalities for reducing the severity of metallic artifacts are nowadays available for both techniques.

The impossibility of MR imaging for patients with MR unsafe implants and devices is a major public health issue. Most of the implants and devices manufactured nowadays are either "MRI-safe" or "MRI-conditional".

Table I lists the factors which lead to inadequate imaging and solutions to obtain correct images.

Conflict of interest: none 


\section{References}

1. Dean Deyle G. The role of MRI in musculoskeletal practice: a clinical perspective. J Man Manip Ther 2011;19:152161.

2. Maniam S, Szklaruk J. Magnetic resonance imaging: Review of imaging techniques and overview of liver imaging. World J Radiol 2010;2:309-322.

3. Kennedy AM, Gilfeather MR, Woodward PJ. MRI of the female pelvis. Semin Ultrasound CT MR 1999;20:214230.

4. Desai MJ, Hargens LM, Breitenfeldt MD, et al. The rate of magnetic resonance imaging in patients with spinal cord stimulation. Spine (Phila Pa 1976) 2015;40:E531-E537.

5. Enders J, Zimmermann E, Rief M, et al. Reduction of claustrophobia during magnetic resonance imaging: methods and design of the "CLAUSTRO" randomized controlled trial. BMC Med Imaging 2011;11:4.

6. ASTM F2503-05: Standard Practice for Marking Medical Devices and Other Items for Safety in the Magnetic Resonance Environment, ASTM International, West Conshohocken, PA, 2005, www.astm.org.

7. Association of Anaesthetists of Great Britain and Ireland, Farling PA, Flynn PA, et al. Safety in magnetic resonance units: an update. Anaesthesia 2010;65:766-770.

8. Expert Panel on MR Safety, Kanal E, Barkovich AJ, et al. ACR guidance document on MR safe practices: 2013. J Magn Reson Imaging 2013;37:501-530.

9. Shellock FG.. Reference Manual for Magnetic Resonance Safety, Implants, and Devices. Los Angeles, CA: Biomedical Research Publishing Group, 2013.

10. Russo RJ, Costa HS, Silva PD, et al. Assessing the Risks Associated with MRI in Patients with a Pacemaker or Defibrillator. N Engl J Med 2017;376:755-764.

11. Stacul F, van der Molen AJ, Reimer P, et al. Contrast induced nephropathy: updated ESUR Contrast Media Safety Committee guidelines. Eur Radiol 2011;21:2527-2541.

12. Quirk M, Ciottone R, Letendre D, Wapner S. Critical person-in-environmental transitions in medical education. Med Teach 1987;9:415-423.

13. Datendorfer K, Amering M, Bankier A, et al. A study of the effect of patient anxiety, perception and equipment on motion artifacts in magnetic resonance imaging. Magn Reson Imaging 1997; 15:301-306.

14. Melendez JC, McCrank E. Anxiety-related reactions associated with magnetic resonance imaging examinations. JAMA 1993;270:745-747.

15. Berg WA, Blume J, Adams AM, et al. Reasons women at elevated risk of breast cancer refuse breast MR imaging screening: ACRIN 6666. Radiology 2010;254:79-87.

16. Dewey M, Schink T, Dewey CF. Claustrophobia during magnetic resonance imaging: cohort study in over 55,000 patients. J Magn Reson Imaging 2007;26:1322-1327.

17. Bangard C, Paszek J, Berg F, et al. MR imaging of claustrophobic patients in an open 1.0T scanner: motion artifacts and patient acceptability compared with closed bore magnets. Eur J Radiol 2007;64:152-157.
18. Koch KM, Lorbiecki JE, Hinks RS, King KF. A multispectral three-dimensional acquisition technique for imaging near metal implants. Magn Reson Med 2009;61:381-390.

19. Lu W, Pauly KB, Gold GE, Pauly JM, Hargreaves BA. SEMAC: slice encoding for metal artifact correction in MRI. Magn Reson Med 2009:66-76.

20. Firmin D, Keegan J. Navigator echoes in cardiac magnetic resonance. J Cardiovasc Magn Reson 2001;3:183-193.

21. Lee VS, Lavelle MT, Rofsky NM, et al. Hepatic MR imaging with a dynamic contrast-enhanced isotropic volumetric interpolated breath-hold examination: feasibility, reproducibility, and technical quality. Radiology 2000;215:365-372.

22. Zaitsev M, Maclaren J, Herbst M. Motion artifacts in MRI: a complex problem with many partial solutions. J Magn Reson Imaging 2015;42:887-901.

23. Jung JW, Kang HR, Kim MH, et al. Immediate hypersensitivity reaction to gadolinium-based MR contrast media. Radiology 2012;264:414-422.

24. Murphy KJ, Brunberg JA, Cohan RH. Adverse reactions to Gadolinium contrast media- a review of 36 cases. Am J Roentgenol 1996;167:847-849.

25. Li A, Wong CS, Wong MK, Lee CM, Au Yeung MC. Acute adverse reactions to magnetic resonance contrast media--gadolinium chelates. Br J Radiol 2006;79:368-371.

26. Becker S, Walter S, Witzke O, Kreuter A, Kribben A, Mitchell A. Application of gadolinium-based contrast agents and prevalence of nephrogenic systemic fibrosis in a cohort of end-stage renal disease patients on hemodialysis. Nephron Clin Pract 2012;121:c91-c94.

27. Lima XT, Alora-Palli MB, Kimball AB, Kay J. Validation of a screening instrument for nephrogenic systemic fibrosis. Arthritis Care Res (Hoboken) 2013;65:637-642.

28. Sosna J, Slasky BS, Bar-Ziv J. Computed tomography in the emergency department. Am J Emerg Med 1997;15:244-247.

29. Tsapaki V, Rehani M, Saini S. Radiation safety in abdominal computed tomography. Semin Ultrasound CT MR 2010;31:29-38.

30. Mettler FA Jr, Huda W, Yoshizumi TT, Mahesh M. Effective doses in radiology and diagnostic nuclear medicine: a catalog. Radiology 2008;248:254-263.

31. National Council on Radiation Protection and Measurements. NCRP Report No. 160, Ionizing radiation exposure of the population of the United States. 2009.

32. Boedeker KL, McNitt-Gray MF. Application of the noise power spectrum in modern diagnostic MDCT: part II. Noise power spectra and signal to noise. Phys Med Biol 2007;52:4047-4061.

33. Huda W, Ogden KM, Samei E, et al. Reconstruction filters and contrast detail curves in CT. Proc. SPIE 6917, Medical Imaging 2008: Image Perception, Observer Performance, and Technology Assessment, 691710 (6 March 2008); doi: 10.1117/12.770530.

34. Mahesh M, Durand DJ. The choosing wisely campaign and its potential impact on diagnostic radiation burden. J Am Coll Radiol 2013;10:65-66.

35. Sistrom CL, Dang PA, Weilburg JB, Dreyer KJ, Rosenthal DI, Thrall JH. Effect of computerized order entry with inte- 
grated decision support on the growth of outpatient procedure volumes: seven-year time series analysis. Radiology 2009;251:147-155.

36. Morris KT, Kavanagh M, Hansen P, Whiteford MH, Deveney K, Standage B. The rational use of computed tomography scans in the diagnosis of appendicitis. Am J Surg 2002; 183:547-550.

37. Barrett JF, Keat N. Artifacts in CT: recognition and avoidance. Radiographics 2004;24:1679-1691.

38. Boas FE, Fleischmann D. Evaluation of two iterative techniques for reducing metal artifacts in computed tomography. Radiology 2011;259:894-902.

39. Andersson KM, Nowik P, Persliden J, Thunberg P, Norrman E. Metal artefact reduction in CT imaging of hip prostheses - an evaluation of commercial techniques provided by four vendors. Br J Radiol 2015;88:20140473.

40. Fleischmann D, Boas FE. Computed tomography - old ideas and new technology. Eur Radiol 2011;21:510-517.

41. Prat-Gonzalez S, Sanz J, Garcia MJ. Cardiac CT: Indications and Limitations. Nucl Med Technol 2008;36:18-24.

42. Kalisz K, Buethe J, Saboo SS, Abbara S, Halliburton S, Rajiah P. Artifacts at Cardiac CT: Physics and Solutions. Radiographics 2016;36:2064-2083.

43. Oliver JH 3rd, Baron RL. Helical biphasic contrast-enhanced CT of the liver: technique, indications, interpretation, and pitfalls. Radiology 1996;201:1-14.

44. Wang CL, Cohan RH, Ellis JH, Caoili EM, Wang G, Francis IR. Frequency, outcome, and appropriateness of treatment of nonionic iodinated contrast media reactions. AJR Am J Roentgenol 2008;191:409-415.

45. Cochran ST, Bomyea K, Sayre JW. Trends in adverse events after IV administration of contrast media. AJR Am J Roentgenol 2001;176:1385-1388.

46. Park HJ, Park JW, Yang MS, et al. Re-exposure to low osmolar iodinated contrast media in patients with prior moderate-to-severe hypersensitivity reactions: A multicentre retrospective cohort study. Eur Radiol 2017;27:2886-2893.

47. Katayama H, Yamaguchi K, Kozuka T, Takashima T, Seez $\mathrm{P}$, Matsuura K. Adverse reactions to ionic and nonionic contrast media. A report from the Japanese Committee on the Safety of Contrast Media. Radiology 1990;175:621-628.

48. Yamaguchi K, Katayama H, Takashima T, Kozuka T, Seez $\mathrm{P}$, Matsuura K. Prediction of severe adverse reactions to ionic and nonionic contrast media in Japan: evaluation of pretesting. A report from the Japanese Committee on the Safety of Contrast Media. Radiology 1991;178:363-367.

49. Fischer HW, Doust VL. An evaluation of pretesting in the problem of serious and fatal reactions to excretory urography. Radiology 1972;103:497-501.

50. Abe S, Fukuda H, Tobe K, Ibukuro K. Protective effect against repeat adverse reactions to iodinated contrast medium: Premedication vs. changing the contrast medium. Eur Radiol 2016;26:2148-2154.

51. Glanc P, O'Hayon BE, Singh DK, Bokhari SA, Maxwell $\mathrm{CV}$. Challenges of pelvic imaging in obese women. Radiographics 2012;32:1839-1862.
52. Ginde AA, Foianini A, Renner DM, Valley M, Camargo CA Jr. The challenge of CT and MRI imaging of obese individuals who present to the emergency department: a national survey. Obesity (Silver Spring) 2008;16:2549-2551.

53. Miller JC, Phil D. Imaging and Obese Patients. Radiology Rounds 2005;3:1-4.

54. Israel GM, Cicchiello L, Brink J, Huda W. Patient size and radiation exposure in thoracic, pelvic, and abdominal $\mathrm{CT}$ examinations performed with automatic exposure control. AJR Am J Roentgenol 2010;195:1342-1346.

55. Ter Haar G. Ultrasonic imaging: safety considerations. Interface Focus 2011;1:686-697.

56. Walas MK, Skoczylas K, Gierblinski I. Errors and mistakes in the ultrasound diagnostics of the liver, gallbladder and bile ducts. J Ultrason 2012;12:446-462.

57. Simmons O, Fetzer DT, Yokoo T, et al. Predictors of adequate ultrasound quality for hepatocellular carcinoma surveillance in patients with cirrhosis. Aliment Pharmacol Ther 2017;45:169-177.

58. Bushberg JT, Seibert JA, Leidholdt EM, Boone JM. The essential physics of medical imaging. $2^{\text {nd }}$ ed. Philadelphia: Lippincott Williams \& Wilkins, 2002.

59. Uppot RN, Sahani DV, Hahn PF, Kalra MK, Saini SS, Mueller PR. Effect of obesity on image quality: fifteen-year longitudinal study for evaluation of dictated radiology reports. Radiology 2006;240:435-439.

60. Modica MJ, Kanal KM, Gunn ML. The Obese Emergency Patient: Imaging Challenges and Solutions. Radiographics 2011;31:811-823.

61. Paladini D. Sonography in obese and overweight pregnant women: clinical, medicolegal and technical issues. U1trasound Obstet Gynecol 2009;33:720-729.

62. Chiorean L, Tana C, Braden B, et al. Advantages and Limitations of Focal Liver Lesion Assessment with Ultrasound Contrast Agents: Comments on the European Federation of Societies for Ultrasound in Medicine and Biology (EFSUMB) Guidelines. Med Princ Pract 2016;25:399-407.

63. Strobel D, Seitz K, Blank W, et al. Contrast-enhanced ultrasound for the characterization of focal liver lesions--diagnostic accuracy in clinical practice (DEGUM multicenter trial). Ultraschall Med 2008;29:499-505.

64. Sturm R, Hattori A. Morbid obesity rates continue to rise rapidly in the United States. Int J Obes (Lond) 2013;37:889891.

65. Calle EE, Rodriguez C, Walker-Thurmond K, Thun MJ. Overweight, obesity, and mortality from cancer in a prospectively studied cohort of U.S. adults. N Engl J Med 2003;348:1625-1638.

66. FDA U.S. Food and Drug Administration. FDA Drug Safety Communication: New warnings for using gadolinium-based contrast agents in patients with kidney dysfunction. Available at: www.fda.gov/Drugs/Drugsafety/ ucm223966

67. Quirk ME, Letendre AJ, Ciottone RA, Lingley JF. Anxiety in patients underogoing MR imaging. Radiology 1989; 170:463-466. 